the preceding month. A review of respiratory care procedures revealed that when mechanical ventilators were serviced between patients, the electronic temperature probes used with servo-controlled humidifiers were wiped with inadequate disinfection. Cultures were taken of casepatient room surfaces, sinks, and ventilator equipment. $S$. maltophilia was recovered from room surfaces, ventilator expiratory circuits, and a temperature sensor that had been kept in ambient air after disinfection. Patients and environmental isolates were examined by randomly amplified polymorphic DNA-polymerase chain reaction.

Three clinical isolates and one environmental isolate had the same profile, which suggests cross-contamination or common source exposure. The outbreak was controlled by adequate disinfection of the temperature sensors. No single epidemic strain was identified, but several observations support the conclusion that the temperature probes contributed to the outbreak.

FROM: Rogues AM, Maugein J, Allery A, et al. Electronic ventilator temperature sensors as a potential source of respiratory tract colonization with Stenotrophomonas maltophilia.J Hosp Infect 2001;49:289-292.

\section{Biofilms and Planktonic Cells of Pseudomonas aeruginosa Have Similar Resistance to Killing by Antimicrobials}

Biofilms are considered to be highly resistant to antimicrobial agents. However, Spoering and Lewis from Northeastern University, Boston, Massachusetts, point out that strictly speaking, this is not the case. Biofilms do not grow any better than planktonic (free floating) cells in the presence of antimicrobials. Biofilms are indeed highly resistant to killing by bactericidal antimicrobials, compared with logarithmic-phase planktonic cells, and therefore exhibit tolerance. It is assumed that biofilms are also significantly more tolerant than stationary-phase planktonic cells. Spoering and Lewis conducted a study to compare, in detail, the tolerance of biofilms versus stationary- and logarithmic-phase planktonic cells to four different antimicrobial agents.

Carbenicillin appeared to be completely ineffective against both stationary-phase cells and biofilms. Killing by this beta-lactam antibiotic depends on rapid growth, and this result confirms the notion of slow-growing biofilms resembling the stationary state. Ofloxacin is a fluoroquinolone antibiotic that kills nongrowing cells, and biofilms and stationary-phase cells were comparably tolerant to it. Most cells in both populations were eradicated at low levels of ofloxacin, leaving a fraction of essentially invulnerable persisters. The bulk of the population in both biofilm and stationary-phase cultures was tolerant to tobramycin. At very high concentrations of tobramycin, a fraction of persister cells became apparent in stationaryphase culture. Stationary-phase cells were more tolerant to the biocide peracetic acid than were biofilms.

In general, stationary-phase cells were somewhat more tolerant than biofilms in all of the cases examined. The authors concluded that, at least for Pseudomonas aeruginosa, one of the model organisms for biofilm studies, the notion that biofilms have greater resistance than do planktonic cells is unwarranted. They further suggest that tolerance to antibiotics in stationary-phase or biofilm cultures is largely dependent on the presence of persister cells.

FROM: Spoering AL, Lewis K. Biofilms and planktonic cells of Pseudomonas aeruginosa have similar resistance to killing by antimicrobials. J Bacteriol 2001;183:6746-6751.

\section{Outbreak of Mycobacterium szulgai Following Laser Eye Surgery}

Laser-assisted in situ keratomileusis (LASIK) is a commonly performed procedure to correct myopia, hyperopia, and astigmatism. After a case of postoperative intracorneal keratitis infection with Mycobacterium szulgai occurred, investigators identified a total of 5 additional patients with this infection of the 52 who had LASIK procedures performed from June 6 to October 24, 2000. All 5 cases were identified among the 18 patients of Dr. A, and no cases were identified among the 34 patients of Dr. B. Two additional patients of Dr. A had had similar corneal lesions, but cultures were not obtained from them. The surgeons' techniques differed only in that Dr. A used a saline lavage that was chilled in a tub of ice, whereas Dr. B used unchilled saline directly from its stock bottle. Extensive environmental cultures were obtained. A culture from the drain of the source ice machine grew $M$. szulgai. Pulse-field gel electrophoresis confirmed this to be identical to all 5 clinical isolates and different from M. szulgai type strain (American Type Culture Collection 35799) and from 3 randomly selected strains.

The investigators concluded that intraoperative contamination from ice water apparently caused the infections. This appears to be the first systematic epidemiologic investigation of an infection cluster following LASIK and the first to link M. szulgai infection with an environmental source. Contaminated ice water from lavage syringes may be a significant source of postoperative ophthalmic infection. Ice water is often contaminated and should not be used in association with surgical procedures.

FROM: Holmes GP, Bond GB, Fader R, Fulcher SF. A chilling experience: a cluster of Mycobacterium szulgai keratitis following laser-assisted in situ keratomileusis. Presented at the 41st Annual Interscience Conference on Antimicrobial Agents and Chemotherapy; December 16-19, 2001; Chicago, Illinois. Abstract no. K-478.

\section{Gram-Negative Bacteria Among Peritoneal Dialysis Patients}

Nasal and pericatheter colonization by Staphylococcus aureus presents an increased risk of peritonitis and exitsite infection for peritoneal dialysis (PD) patients. PerezFontan and colleagues from Hospital Juan Canalejo, A Coruna, Spain, conducted a study to examine the inci- 